\title{
The involvement of Central Nervous System and sequence variability of Severe Adult Respiratory Syndrome - Coronavirus-2 revealed in autopsy tissue samples: a case report.
}

\section{Lis Høy Marbjerg}

Department of Virus and Microbiological Special Diagnostics, Division of Infectious Disease Preparedness, Statens Serum Institut, Artillerivej 5, Copenhagen S, Denmark https://orcid.org/00000001-9259-3525

\section{Christina Jacobsen}

Section of Forensic Pathology, Department of Forensic Medicine, University of Copenhagen, Copenhagen, Denmark https://orcid.org/0000-0001-7257-0772

\section{Jannik Fonager}

Department of Virus and Microbiological Special Diagnostics, Division of Infectious Disease Preparedness, Statens Serum Institut, Artillerivej 5, Copenhagen S, Denmark https://orcid.org/00000003-1688-1482

\section{Claus Bøgelund}

Section of Forensic Pathology, Department of Forensic Medicine, University of Copenhagen, Copenhagen, Denmark

\section{Morten Rasmussen}

Department of Virus and Microbiological Special Diagnostics, Division of Infectious Disease Preparedness, Statens Serum Institut, Artillerivej 5, Copenhagen S, Denmark https://orcid.org/00000001-7491-4137

\section{Anders Fomsgaard}

Department of Virus and Microbiological Special Diagnostics, Division of Infectious Disease Preparedness, Statens Serum Institut, Artillerivej 5, Copenhagen S, Denmark https://orcid.org/00000003-4304-0603

\section{Jytte Banner}

Section of Forensic Pathology, Department of Forensic Medicine, University of Copenhagen, Copenhagen, Denmark https://orcid.org/0000-0002-7559-1285

\section{Veronika Vorobieva Solholm Jensen ( $\nabla$ veronika.v.vorobieva@gmail.com )}

Department of Virus and Microbiological Special Diagnostics, Division of Infectious Disease Preparedness, Statens Serum Institut, Artillerivej 5, Copenhagen S, Denmark https://orcid.org/00000001-8685-1882 


\section{Case Report}

Keywords: complete autopsy, COVID-19, whole-genome sequencing of SARS-CoV-2, neuroinvasion

Posted Date: August 19th, 2020

DOl: https://doi.org/10.21203/rs.3.rs-61471/v1

License: (c) (i) This work is licensed under a Creative Commons Attribution 4.0 International License. Read Full License 


\section{Abstract}

Background: The case presented here illustrates that interdisciplinary teamwork can be essential for the understanding of the COVID-19 disease presentation and enlightening of the pathophysiology.

Case presentation: A 60-years-old overweight woman without any comorbidities was found dead in her apartment after 14 days of home isolation due to suspicion on the Coronavirus disease 2019 (COVID-19). She had reported symptoms of tachycardia, fever, and increasing respiratory difficulty one day before her death. Due to the Danish legal act on sudden deaths a forensic autopsy was performed including a thorough examination and biosampling. The results of the forensic autopsy displayed sever densified, almost airless, firm lungs, and an unspecific reactive minimal focal perivascular inflammation consisting of macrophages of the brain tissue. The final diagnosis, COVID-19 with involvement of the central nervous system was established by use of the RT-RNA analysis on cerebrospinal fluid, as well as by serologic detection of the specific antibodies for SARS-CoV-2 in cerebrospinal fluid and serum. The genetic analysis displayed a $2 \%$ variation between SARS-CoV-2 isolates recovered from the tracheal sample, cerebrospinal fluid, and tissues from both lungs.

Conclusion: The combination of all available results revealed that the cause of death was COVID-19 with severe pulmonary disease and neuroinvasion, as well as renal affection resulting in hyponatremia. To our knowledge, it was not shown previously that neuroinvasion could be confirmed by the detection of specific antibodies for SARS-CoV-2 and SARS-CoV-2 specific RNA in cerebrospinal fluid. This case supports hypotheses that SARS-CoV-2 may cause central nervous system infection. The genetic distinction between SARS-CoV-2 isolates was done by whole-genome sequencing, where the isolate recovered from the cerebrospinal fluid was the most different.

\section{Background}

Coronavirus disease 2019 (COVID-19) is an infection caused by Severe Acute Respiratory Syndrome Coronavirus 2 (SARS-CoV-2) which arose in Wuhan Hubei Province, China in late 2019 ${ }^{1}$. SARS-CoV-2 is a relatively large single-stranded RNA virus, that is, closely related to SARS-CoV ${ }^{1}$. It has since spread and caused more than 483.217 deaths worldwide (https://gisanddata.maps.arcgis.com/apps/opsdashboard/index.html). By the middle of July 2020, a total of 611 cases of death were confirmed in Denmark by the Health Authorities with $4.7 \%$ of deaths to a total number of confirmed infected cases (https://www.ssi.dk/sygdomme-beredskab-ogforskning/sygdomsovervaagning/c/covid19-overvaagning). The National Danish Register includes deaths recorded within 30 days of COVID-19 infection detected and the COVID-19 is not necessarily being the main cause of death.

An autopsy is a diagnostic tool to elucidate the pathogenesis of the COVID-19, and to discover SARSCoV-2's pathogenic characteristics. In this case, a forensic autopsy was performed due to the Danish legal act on sudden death. The autopsy was performed according to ISO 17020 standards and included a 
whole body post-mortem computer tomography (PMCT) prior to the external and internal examination, the thorough biosampling, and the additional supplementary toxicological and histopathological tests, as well as extensive microbiological analyses, were performed. According to recently published autopsy studies, the primary cause of death in individuals who died of COVID-19 was a respiratory failure with exudative diffuse alveolar damage $\mathrm{e}^{3}$. Additionally, it has been revealed that the leading causes of death in COVID-19-positive patients are bronchopneumonia, massive pulmonary embolism, and alveolar haemorrhage $e^{3,4}$.

Symptoms such as ataxia, seizures, as well as a loss of smell and taste have led to the hypothesis that the virus may be also neurotropic, possible through ACE2-receptors that are expressed in the brain ${ }^{5}$. It has also been suggested that neuroinvasion of SARS-CoV-2 may play a role in the respiratory failure of COVID-19 patients. Here we describe a woman with probable central nervous system (CNS) involvement due to COVID-19.

\section{Case Presentation}

\section{Patient information.}

A 60-years-old overweight woman (Body Mass Index 28.5), without prior conditions, was found dead in her bed by her partner. The circumstantial information in the police report revealed that she had been in home-isolation for 14 days due to suspicion of COVID-19 infection. In the days preceding her death, she reported symptoms of high puls (around 100 beats per minute), fever, and increasing respiratory difficulty to her family. The partner had been in contact with the deceased continually during the 14 days and neighbours had done the grocery shopping, which was left in front of her main door. During the last days, the decendent had been in telephone contact with her primary physician as well as the emergency medical service. She had an appointment with her primary physician, which was meant to take place the day after she was found dead. Clinical records were checked for pre-existing medical conditions and medications and none were reported.

Diagnostic assessment.

Post-mortem CT-scan (PMCT). The lungs were uniformly consolidated bilaterally with air entrapment (Figure $1 \mathrm{~A}$ and $\mathrm{B}$ ). The abdominal organs and the brain were without significant changes, apart from unspecific diffuse moderate edema of the brain, a calcified isolated process related to the pericardium and subtle calcification of the mitral valves.

Autopsy and histology.

The autopsy revealed heavily densified, almost airless, firm lungs with a total weight of 1460 grams without signs of purulent secretion. Pleura was without fibrin deposits, but the mucosa was hyperaemic. Tracheobronchial lymph nodes were enlarged. The brain was edematous weighing 1512 grams (normal 
weight 1250 grams $+/-20$ grams $^{8}$ ), with slight flattening of the gyri, unremarkable arachnoid, gray and white matter, and without signs of inflammation. The kidneys showed signs of acute shock.

The histological examination was performed according to standard protocols ${ }^{6,7}$ and supplementary immunohistochemistry was performed for structural cell types comprising cytokeratin 7 (CK7), Thyroid transcription factor 1 (TTF-1), Platelet/endothelial cell adhesion molecule-1 (CD31), Smooth Muscle actin (SMA) and inflammatory markers of T-lymphocytes (CD3, CD4, CD8, FOX-P3), B-lymphocytes (CD20), plasma cells (CD79a, CD138, IgG, IgM), epithelial cells (HLA-DR) and complement fractions (C4d and C3d).

The histopathology of the lung tissue revealed universally severe diffuse alveolar damage ranging from characteristic acute changes to more chronic changes (Figure $1 \mathrm{C}-\mathrm{F}$ ). There were no visual signs of viral inclusions. The endothelium in the vasculature was severely hypoplastic with necrotizing, thrombotic microangiopathy. The complement fraction $\mathrm{C} 4 \mathrm{~d}$ (Figure $1 \mathrm{G}$ and $\mathrm{H}$ ) was expressed in the endothelium. Additionally, the cellular immunohistochemistry showed a dominance of CD3 positive T-lymphocytes and only a few CD20 positive B-lymphocytes. The T-lymphocytes were predominantly CD 4 positive, with fewer CD8 and only very few FOX P3 cells. The inflammatory substrate was dominated by CD 68 positive macrophages and plasma cells with severe deposition of IgM, IgG as well as HLA-DR in the epithelial, endothelial, and inflammatory cells.

Tissue from the CNS comprised cerebrum with the motoric cortex, basal ganglia, hippocampus, pons, and cerebellum. The meninges were sparsely represented. The tissue showed an unspecific reactive minimal focal perivascular inflammation consisting of macrophages. There was no edematous change, vasculitis, microthrombi, and no deposition of complement fractions.

Myocardial tissue was with unspecific subtle edema. Tissue from the liver, spleen, lymph nodes, kidneys, bone marrow, and suprarenal glands showed relevant unspecific hyperplastic changes and slight acute tubular necrosis in the kidneys.

Toxicology. Toxicologic analyses of blood and vitreous fluid for medication, illicit drugs, alcohol, diabetesrelated analyses, and sodium as well as pottasium concentration were performed. The results showed a treatment-related concentration of paracetamol. The post-mortem electrolyte concentrations showed hyponatremia $\left(103 \mathrm{mmol} / \mathrm{L}\right.$, defined $<134 \mathrm{mmol} / \mathrm{L}^{9}$ ) while the potassium concentration was as expected in post-mortem material $(21 \mathrm{mmol} / \mathrm{L})$.

RNA-extraction. RNA extraction was performed in the ISO Certified routine PCR diagnostic reference laboratory using automated nucleic acid extraction (MagNA Pure 96, Roche) according to manufacture recommendations. The detection of SARS-CoV-2 was performed by real-time reverse transcription PCRbased assay as described previously ${ }^{10}$ performed on post-mortem isolates. SARS-CoV-2 was identified in the CSF with cycle threshold (Ct) at 38.23, lung tissue from both left and right lung with Ct-values at 28.18 and 25.10 , respectively, and in the tracheobronchial specimen with $\mathrm{Ct} 26.76$. The blood specimen and the heart tissue specimen were negative for SARS-CoV-2. 
Multiplex RT-PCR. Multiplex RT-PCR-based assay for seventeen different respiratory tract virus (influenza type $A$ and $B$, respiratory syncytial virus type $A$ and $B$, metapneumovirus, parainfluenzavirus type 1, 2, 3 and 4, adenovirus, rhinovirus, enterovirus, parechovirus and coronavirus OC43, 229E, NL63, HKU1), was performed. Tissue samples from the heart and both lungs, as well as tracheobronchial secretion, blood, and CSF were analysed by this assay. None of the viruses was detected in any of the samples.

Culturing. The culturing of the CSF showed the growth of Staphylococcus capitis. Besides, the growth of Bacillus cereus was identified of tissue from the right lung. Both of findings were interpreted as either postmortem bacterial migration or contamination.

SARS-CoV-2 specific antibodies testing. Two enzyme-linked immunosorbent assays (ELISA), iFlash 1800 Chemiluminescence Immunoassay Analyser (YHLO Biotech Co., Shenzhen, China) and the Wantai SARSCoV-2 Total Antibody ELISA (Beijing Wantai Biological Pharmacy Enterprise, Beijing, China), were performed for investigation of the serological profile of the patient according to the manufacturer's instructions and, as it was described earlier ${ }^{11}$. We examined serum and CSF with both methods. These tests are CE-marked, but not validated for the detection of antibodies in CSF.

Sequencing of SARS-CoV-2. Total RNA was converted to CDNA with Superscript IV First-Strand Synthesis Kit (Thermo) using $11 \mu \mathrm{l}$ of extracted RNA and random hexamers according to the manufacturer's specifications. PCR based amplification of the viral genome was performed according to the ARTIC protocol using two separate multiplex primer pools reactions of the version 3 release of the primer set (https://artic.network/ncov-2019). Nanopore Sequencing libraries were prepared according to the Artic protocol and loaded onto an FLO-MIN106D Type R9.4.1 flow cell and sequenced on the MinION device (Oxford Nanopore, UK). Illumina libraries were prepared using the Nextera XT DNA Library Prep Kit (Illumina, CA). Paired-end sequencing was performed on the MiSeq platform (Illumina, CA).

Sequence analysis was performed in CLC genomics workbench 20.0.4 with the Long read support (Beta) plug-in installed for the import and mapping of Oxford Nanopore reads. Demultiplexed Reads were imported as Oxford Nanopore reads and paired Illumina reads respectively. Reads were trimmed against the V3 primer set $(n=218)$ to remove primer sequences from subsequent analysis. Oxford Nanopore reads and Illumina reads were mapped individually to the MT135044 SARS-CoV-2 reference sequence at CLCs default settings. To enhance the fraction of the genome sequenced and read-coverage (depth), Nanopore and Illumina mappings were merged into one for sequences from each of the 4 samples. This mapping had the following number of mapped reads, fraction (\%) of the genome sequenced and average read coverages: Spinal: 101181, 98\%, 294.12; Tracheal: 346914, 99\%, 1041.55; L lung: 5044442, 99.9\%, 14041.95; R lung: 4431907, 100\%, 12191.83. Consensus sequences were generated from the merged read mappings both at any coverage and at a minimum coverage of 5 reads and inserting $\mathrm{N}$ as a symbol at sequence positions not covered sufficiently. Sequence reads from the four different samples were individually aligned with the reference sequence and manually inspected and corrected in BioEdit 7.2.5 $5^{12}$. Only inter-sample variants observed in the MiSeq mapping covered by $\geq 5$ reads and also observed in the Nanopore mapping were included in the consensus sequences. Non-resolved variant sites were marked 
with an $\mathrm{N}$ in the consensus sequence and excluded from further analysis. After this curation step, the fraction of the genome sequenced (in \%) were: Spinal: 92.9\%; Tracheal: $98.9 \%$; L lung: 99.9\%, and R lung: $100 \%$.

Genotyping of SARS-CoV-2 samples. The clade designation system (https://nextstrain.org/ncov/global) was used by downloading the Nextstrain Metadata set on the $28^{\text {th }}$ of May 2020. From this Metadata list, 27 sequences, representing all major SARS-CoV-2 clades (A1a, A2, A2a, A3, A6, B, B1, B2, and B4; only clades represented by at least 10 sequences were included) were selected along with the Wuhan-Hu-1 sequence (NC_045512). These 28 reference sequences were aligned with four patient sequences (named after their anatomical origin: L Lung, R Lung, Tracheal, and Spinal) in Mafft

(https://mafft.cbrc.jp/alignment/server/index.html). Phylogenetic analysis was performed in Mega 10 ${ }^{13-}$ 15 using the Maximum Likelihood statistical method and the Hasegawa-Kishino-Yano model with a Gamma parameter of 2 and 100 bootstrap replicates and complete deletion of any gaps or missing data. The phylogenetic analysis (Figure 2) showed that all four virus samples belonged to the A2a clade.

Sequences from three of the samples (Spinal, Tracheal, and R lung) had a dominant SNP at three sites in the ORF1ab gene (Table 1), two of which (R lung and Tracheal) were both mixed mutations. In sequences from the R lung, the SNP (T) was present in $66 \%$ of the reads while the $G$ shared with the sequences from other samples was present in $33 \%$ of reads. Two minor variants, $C$ and $A$ constituted $0.01 \%$ and $0.06 \%$ of the reads and probably is most likely background seen at the high coverage observed at this site. The SNPs in the spinal and the Tracheal samples resulted in changes in the encoded amino acid (Table 2). The majority of viral sequences in the spinal sample encoded a Glycine $(G)$ instead of a Serine $(S)$ in the 3-C proteinase gene and the majority of viral sequences in the tracheal sample encoded a $\mathrm{V}$ aline $(\mathrm{V})$ instead of a Glycine $(G)$ in the NSP11 encoding gene.

\section{Discussion And Conclusions}

The arrangement of all available results revealed that the cause of death was COVID-19 with severe pulmonary disease and neuroinvasion, as well as renal affection resulting in hyponatremia. A previous report found that $36.4 \%$ of COVID-19 patients had neurological manifestations and that the rate of neurological symptoms was higher in patients with severe infection ${ }^{16}$. However, in a recent report from Japan, a 24-year-old man was diagnosed with COVID-19 related meningitis/encephalitis, where specific RNA-SARS-CoV-2 was detected in CSF, while it was not detected in the nasopharyngeal swabs ${ }^{17}$. In our case, specific RNA-SARS-CoV-2 was detected in the tracheal sample, CSF, and tissue from both lungs. CNS-infection in relation to COVID-19 is further supported, by a report where the presence of the virus in neural and capillary endothelial cells, in the frontal lobe of a patient with COVID-19 infection and neurological symptoms, were documented by electron microscopy ${ }^{18}$. The microscopic examination of the brain revealed no signs of encephalitis, microthrombi, or inflammation, which is in keeping with Menter $T$ et $a l^{3}{ }^{3}$, who, apart from unspecific microscopic findings, registered a low viral load in neurological tissue. 
Phylogenetic analysis showed that all four samples belonged to the same A2a clade. Most European SARS-CoV-2-sequences on Nextstrain as per $28^{\text {th }}$ of May 2020 belong to this clade (3177 of 4082; $77.8 \%$ ). In Denmark, this clade is the most dominant (206 of 208; 99.03\%) (https://nextstrain.org/ncov/europe). The phylogenetic analysis showed that the spinal fluid sample differed the most from the other three samples. The reason for this is unknown, but we hypothesize that this could be due to the selection of more fit viruses and/or founder effects/bottlenecks when moving between compartments, as has been demonstrated previously for other viruses ${ }^{19}$.

When it comes to sequencing/PCR-results you always have to consider the possible error sources. Also for the spinal sample, $7.9 \%$ of the genome was not covered or the quality was too low for analysis, which might have lead to some mutations not being detected. However, since this is post-mortem material, which cannot be recovered once it is used, and since we used state of the art methods, this limitation cannot be further addressed with currently available methods.

In the above-described case, the patient had been ill for 14 days before her death and this timeline is supported by the finding of SARS-CoV-2 specific antibodies. As has been found by others ${ }^{20}$, also the microscopic changes in the lung tissue, in this case, were consistent with the timeline with the representation of severe diffuse alveolar damage in the early phase, but also in an organizing and proliferative intermediary phase, indicating a disease process lasting around one to two weeks with a continuing severe acute component affecting both the pulmonary parenchyma, respiratory epithelium, and vasculature $20,21,3$.

Biochemical analysis, in this case, showed severe hyponatremia, which is suspected to be the result of several components: kidney affection with tubular necrosis and possibly increased water intake during the disease. The deceased was isolated in the weeks before her death and information regarding food and water intake in the last days is not available. It is concluded, that hyponatremia is a secondary result of a prolonged disease course.

To our knowledge, this is the first report were both specific antibodies for SARS-CoV-2 and SARS-CoV-2 specific RNA were detected in CSF. However, the detection of specific antibodies for SARS-CoV-2, in CSF is not validated and the clinical significance is unclear. To conclude, this case supports hypotheses that SARS-CoV-2 may cause central nervous system infection. The pathogenesis, incidence, and clinical impact, as well as the detection of specific antibodies for SARS-CoV-2 in CSF, need to be investigated further.

\section{List Of Abbreviations}

ACE2-Angiotensin-converting enzyme 2

ARTIC-novel coronavirus bioinformatics protocol cDNA-complementary DNA 
Cd3-complement factor 3d

CD31-platelet / endothelial cell adhesion molecule -1

Cd4-complement factor $4 d$

CE-marked-Conformitè Europëenne-marked

CK-cytokeratin

CLC-Genomics Workbench desktop software

CNS-Central Nervous System

COVID-19-Coronavirus disease 2019

CSF-cerebrospinal fluid

Ct-cycle threshold

G-Glycine

ELISA-enzyme-linked immunosorbent assay

HLA-DR-human leukocyte antigens complex

IgG-Immunoglobulin class $\mathrm{G}$

IgM-Immunoglobulin class $\mathrm{M}$

PMCT-post-mortem computer tomography

RT-PCR-reverse transcription-polymerase chain reaction

SARS-CoV-2-Severe acute respiratory syndrome coronavirus 2

S-Serine

SMA-Smooth Muscle Actin

SNP-single nucleotide polymorphism

TTF-1-Thyroid Transcription Factor-1

V-Valine

\section{Declarations}


Ethics approval and consent to participate

The written consent from the next-of-kin, as well as consent from the police authorities, was obtained.

Consent for publication

Not applicable

\section{Availability of data and materials}

The data and materials are available on request from the corresponding author (Veronika Vorobieva Solholm Jensen, Dept. of Virus and Microbiological Special Diagnostics, Statens Serum Institute; Artillerivej 5, DK-2300 Copenhagen S, Denmark. Email:veronika.v.vorobieva@gmail.com), but restrictions apply under licence for the current study.

\section{Declaration of competing interest}

The authors declare that they have no conflict of interest.

\section{Funds}

All authors were funded by their departments. VVSJ and JB are involved with the project supported by Lundbeck fund. The funds had no role in study design, data collection, and analysis, decision to publish, or preparation of the manuscript.

\section{Authors' contributions}

VVSJ and JB designed the study. JB, CB, and $C J$ contributed to the collection of the samples. $C J, J F, C B$, MR carried out laboratory analysis. LHM, CJ, JF, CB, MR, AF, JB, and VVSJ carried out data analysis. All authors wrote the manuscript and approved its publication.

\section{Acknowledgments}

The authors acknowledge the staff of the Clinical microbiological laboratory in the University Hospital, Rigshospitalet, the PCR-laboratory and Laboratory for Serology, Virus and Microbiological Special Diagnostics and Infectious Disease Preparedness, Statens Serum Institute, and Section of Forensic Pathology, Department of Forensic Medicine, University of Copenhagen for excellent laboratory support. Finally, we would like to thank the next-of-kin for giving their consent for publication of this case report.

\section{References}

1.Chan, J. F. W. et al. Genomic characterization of the 2019 novel human-pathogenic coronavirus isolated from a patient with atypical pneumonia after visiting Wuhan. Emerg. Microbes Infect. 9, 221-236 (2020). 
2.Zhou, F. et al. Clinical course and risk factors for mortality of adult inpatients with COVID-19 in Wuhan, China: a retrospective cohort study. Lancet 395, 1054-1062 (2020).

3.Menter, T. et al. Post-mortem examination of COVID19 patients reveals diffuse alveolar damage with severe capillary congestion and variegated findings of lungs and other organs suggesting vascular dysfunction. Histopathology (2020) doi:10.1111/his.14134.

4.Wichmann, D. et al. Autopsy Findings and Venous Thromboembolism in Patients With COVID-19. Ann. Intern. Med. 25, (2020).

5.Baig, A.M., Khaleeq, A., Ali, U. \& Syeda, H. Evidence of the COVID-19 Virus Targeting the CNS: Tissue Distribution, Host-Virus Interaction, and Proposed Neurotropic Mechanisms. ACS Chemical Neuroscience (2020) doi:10.1021/acschemneuro.0c00122.

6.Berry, G. J. et al. The 2013 international society for heart and lung transplantation working formulation for the standardization of nomenclature in the pathologic diagnosis of antibody-mediated rejection in heart transplantation. Journal of Heart and Lung Transplantation vol. 32 1147-1162 (2013).

7.Wallace, W. D. et al. Banff study of pathologic changes in lung allograft biopsy specimens with donorspecific antibodies. J. Hear. Lung Transplant. 35, 40-48 (2016).

8.AJ Connolly, W. F. et al. Autopsy pathology - A manual and atlas. (Elsevier, 2015).

9.DeVita, MV, Gardenswartz, M. et al. Incidence and Etiology of Hyponatremia in an Intensive Care Unit PubMed. Clin. Nephrol. 34, 163-166 (1990).

10.Corman, V. M. et al. Detection of 2019 novel coronavirus (2019-nCoV) by real-time RT-PCR. Eurosurveillance 25, 1 (2020).

11.Lassaunière, R. et al. Evaluation of nine commercial SARS-CoV-2 immunoassays. medRxiv 2020.04.09.20056325 (2020) doi:10.1101/2020.04.09.20056325.

12.Hall TA. BioEdit: a user-friendly biological sequence alignment editor and analysis program for Windows 95/98/NT. Nucl. Acids. Symp. Ser., 95-98. (1999).

13.Hasegawa, M., Kishino, H. \& Yano, T. aki. Dating of the human-ape splitting by a molecular clock of mitochondrial DNA. J. Mol. Evol. 22, 160-174 (1985).

14.Kumar, S., Stecher, G., Li, M., Knyaz, C. \& Tamura, K. MEGA X: Molecular evolutionary genetics analysis across computing platforms. Mol. Biol. Evol. 35, 1547-1549 (2018).

15.Felsenstein, J. CONFIDENCE LIMITS ON PHYLOGENIES: AN APPROACH USING THE BOOTSTRAP. Evolution (N. Y). 39, 783-791 (1985). 
16.Mao, L. et al. Neurologic Manifestations of Hospitalized Patients With Coronavirus Disease 2019 in Wuhan, China. JAMA Neurol. (2020) doi:10.1001/jamaneurol.2020.1127.

17.Moriguchi, T. et al. A first case of meningitis/encephalitis associated with SARS-Coronavirus-2. (2020) doi:10.1016/j.ijid.2020.03.062.

18.Paniz-Mondolfi, A. et al. Central Nervous System Involvement by Severe Acute Respiratory Syndrome Coronavirus -2 (SARS-CoV-2). J. Med. Virol. jmv.25915 (2020) doi:10.1002/jmv.25915.

19.Brese, R. L. et al. Ultradeep single-molecule real-time sequencing of HIV envelope reveals complete compartmentalization of highly macrophage-tropic R5 proviral variants in brain and CXCR4-using variants in immune and peripheral tissues. J. Neurovirol. 24, 439-453 (2018).

20.Polak, S. B., Van Gool, I. C., Cohen, D., Von Der Thüsen, J. H. \& Van Paassen, J. A systematic review of pathological findings in COVID-19: a pathophysiological timeline and possible mechanisms of disease progression. Mod. Pathol. doi:10.1038/s41379-020-0603-3.

21.Barton, L. M., Duval, E. J., Stroberg, E., Ghosh, S. \& Mukhopadhyay, S. COVID-19 Autopsies, Oklahoma, USA. Am. J. Clin. Pathol. doi:10.1093/AJCP/AQAA062.

\section{Tables}

Table 1. Comparison of the nucleotide diversity in the four isolates of SARS-CoV-2 recovered from the four different tissue samples.

\begin{tabular}{|llll|}
\hline & \multicolumn{4}{l}{ Nucleotide position on NC_045512 } \\
\hline Sample ID & 787 & 10097 & 19553 \\
\hline Spinal & $\mathrm{g}$ & $\mathrm{g}$ & $\mathrm{g}$ \\
\hline Tracheal & $\mathrm{g}$ & $\mathrm{a}$ & $\mathrm{t}$ \\
\hline L Lung & $\mathrm{g}$ & $\mathrm{a}$ & $\mathrm{g}$ \\
\hline R lung & $\mathrm{t}$ & $\mathrm{a}$ & $\mathrm{g}$ \\
\hline Supporting MiSeq reads & $\mathrm{T}: 33195 / \mathrm{G}: 16666 / \mathrm{C}: 30 / \mathrm{A}: 8$ & $\mathrm{G}: 23$ & $\mathrm{~T}: 55$ \\
\hline Supporting Nanpore reads & $\mathrm{T}: 117 / \mathrm{G}: 77 / \mathrm{C}: 4 / \mathrm{A}: 1$ & $\mathrm{G}: 4$ & $\mathrm{G}: 4 / \mathrm{T}: 2$ \\
\hline
\end{tabular}

Table 2. Variations of the amino acids in the four isolates of SARS-CoV-2 recovered from the four different tissue samples. 


\begin{tabular}{|lll|}
\hline & \multicolumn{2}{l|}{ Amino acid position on the ORF1ab gene } \\
\hline & 3278 & 6430 \\
\hline Spinal & G & G \\
\hline Tracheal & S & V \\
\hline L lung & S & G \\
\hline R lung & S & G \\
\hline
\end{tabular}

Figures

A.

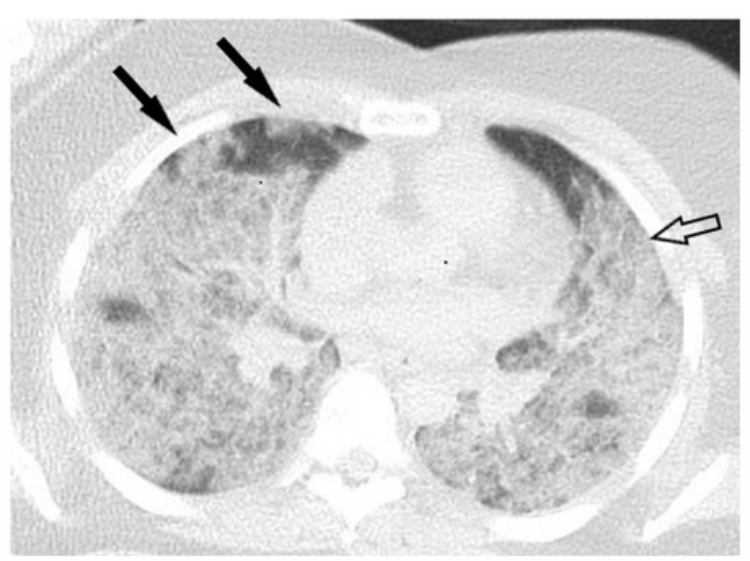

B.

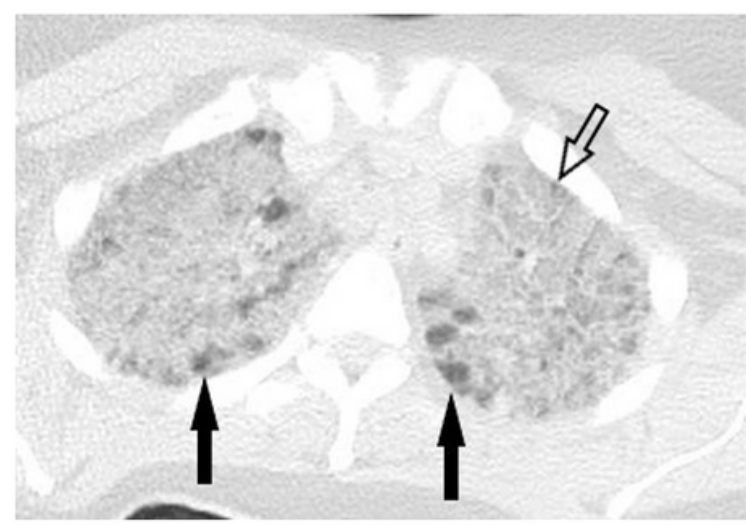

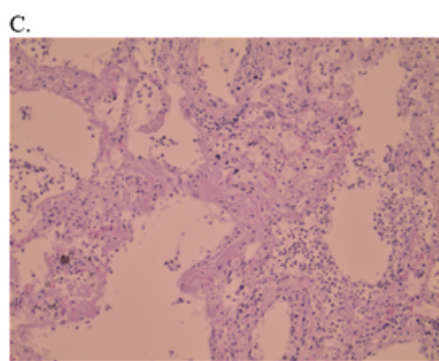

D.
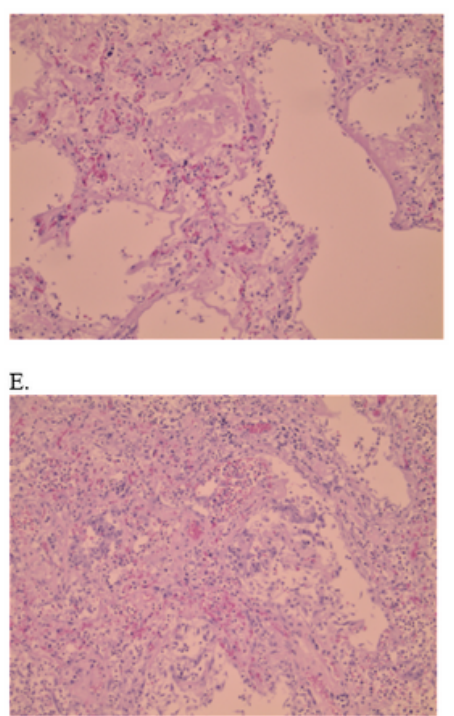

F.

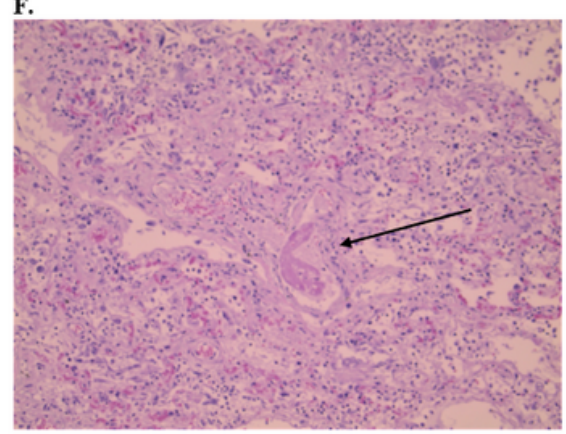

G.

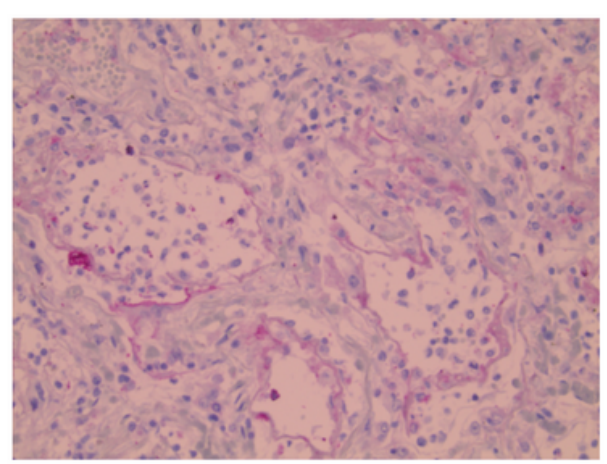

H.

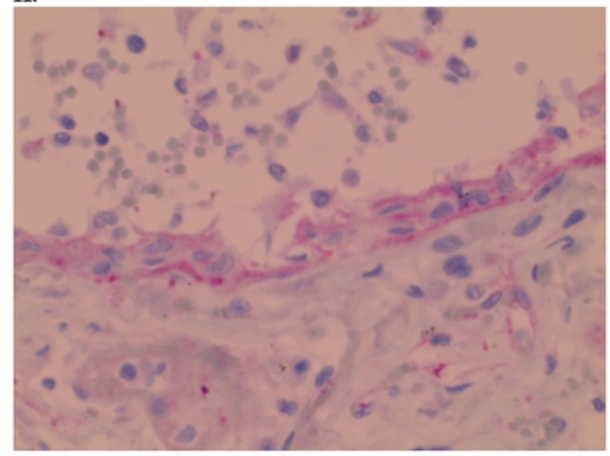

Figure 1 
A. Axial post-mortem computer tomography scan of the consolidated lungs with peripheral ground glass opacities (solid arrows) and crazy paving (open arrow). B. Axial post-mortem computer tomography scan of the consolidated lungs with air entrapment (solid arrows) and crazy paving (open arrow). C-F. Microscopic pulmonary findings of diffuse alveolar damage showing a spectrum of acute exudative lesions ( $C-D)$ to a more consolidated phase with proliferation and organization (E), including a thrombus in the vasculature (F, arrow) (Hematoxylin and eosin staining, 200x). G-H. Microscopic immunohistochemical findings showing the deposition of C4d complement fraction in the alveolar structures (G) and microvasculature (H) (IHC for C4d stain; 200x). 


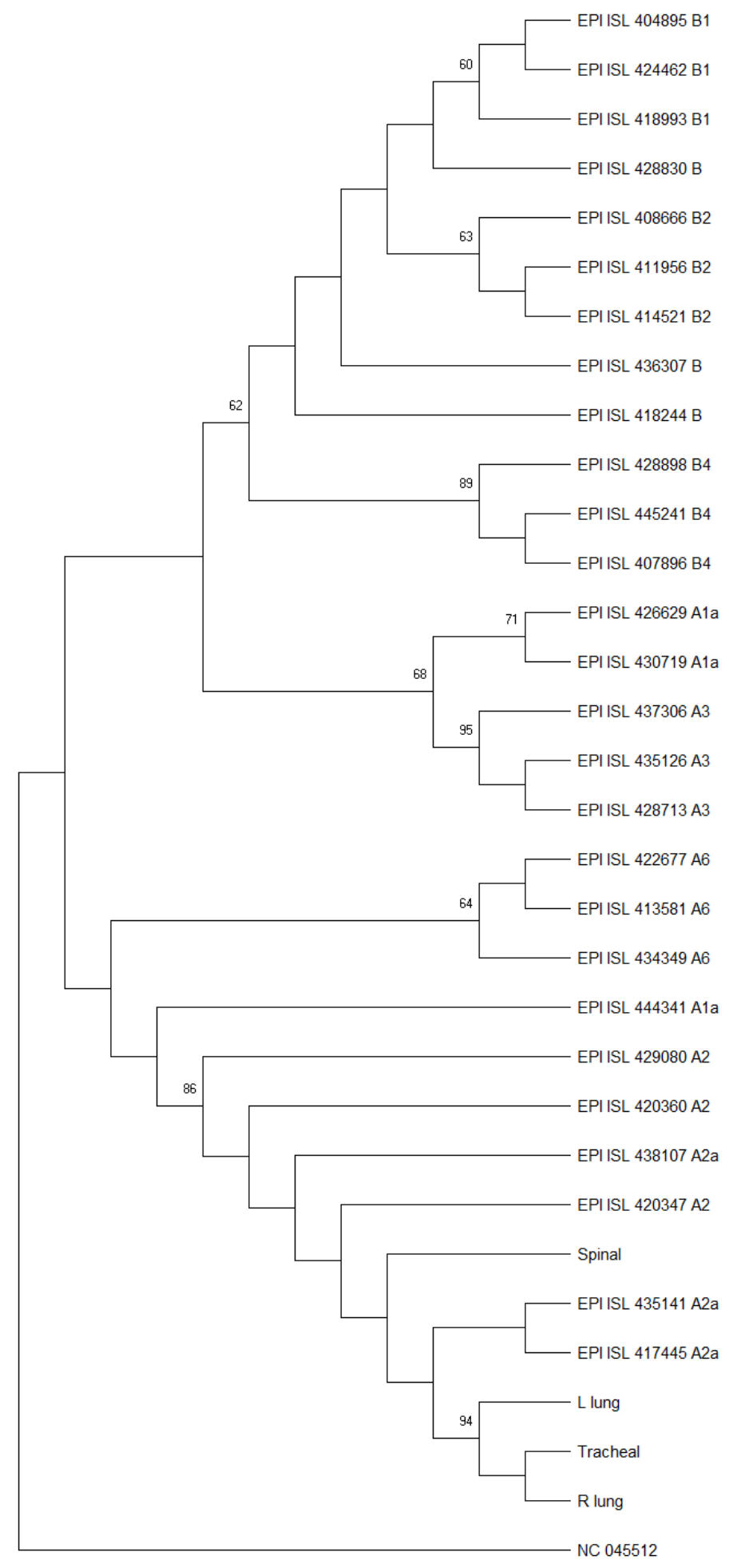

\section{Figure 2}

Phylogenetic analysis of patient sequences. Phylogenetic tree rooted with the Wuhan-Hu-1 sequence (NC_045512) and GISAID sequences, shown for example EPI ISL 420347 (GISAID identifier) A2 (Nextstrain clade) and the four patient sequences (Spinal, Tracheal, R lung, and L lung). 